PROCEEDINGS OF THE

AMERICAN MATHEMATICAL SOCIETY

Volume 31, No. 1, January 1972

\title{
A REMARK ON "PAIRS" OF REGULAR FUNCTIONS
}

\author{
JAMES A. JENKINS
}

Abstract. See $\$ 1$.

1. Quite some years ago the author proved that for the class $C$ of Bieberbach-Eilenberg functions [2] there holds the following exact bound: if $f(z) \in C,|z|=r, r<1,|f(z)| \leqq r\left(1-r^{2}\right)^{-1 / 2}$ with equality only for the functions $\pm f_{r}\left(z e^{i \theta}\right)$ at the respective points $z=i e^{-i \theta} r, \theta$ real, where $f_{r}(z)=\left(1-r^{2}\right)^{1 / 2} z(1+i r z)^{-1}$. Recently Aharonov [1] has shown that if $F, G$ are functions regular in $|z|<1$ with $F(0)=G(0)=0$ and such that $F(z) G(\zeta) \neq 1$ for arbitrary $z, \zeta$ with $|z|,|\zeta|<1$ then $|F(z) G(\zeta)| \leqq$ $|z||\zeta|\left(1-|z|^{2}\right)^{-1 / 2}\left(1-|\zeta|^{2}\right)^{-1 / 2}$. It should be remarked that various authors have studied the equivalent situation where $f$ is regular for $|z|<1$ with $f(0)=0, F$ meromorphic in $|\zeta|>1$ with a pole at the point at infinity, and the respective images have no common point also by the use of the area principle. (See [4] where moreover further references are given.) In the present instance while Aharonov asserts that his bound is sharp this is true only in the crude sense that there are some pairs of values of $|z|,|\zeta|$ (specifically $|z|=|\zeta|$ ) for which equality occurs. A more complete answer to the problem is attained by finding a function $H(s, t)$ defined for $(s, t) \in(0,1) \times(0,1)$ such that $|F(z) G(\zeta)| \leqq H(|z|,|\zeta|)$ where equality is attained for suitable functions for every choice of values of $|z|,|\zeta|, 0<|z|,|\zeta|<1$. This can be done by the author's original method given in [2]. It should be reiterated with regard to that paper that the result initially mentioned was there derived by a method of Teichmüller rather than by use of symmetrization because at that time there was no known equality result for the latter. This was supplied within several years [3] so that we now use the symmetrization argument which is more natural.

2. Let $E_{r}, 0<r<1$, denote the doubly-connected domain obtained by slitting the unit circle along the segment from 0 to $r$ and let $v(r)$ be its module. Let $\Delta_{K}, 0<K<\infty$, denote the doubly-connected domain obtained by slitting the plane along the segment from 0 to 1 and along the negative real axis to the left of $-K$ and let $\mu(K)$ be its module. (This is a

Received by the editors February 11, 1971.

AMS 1970 subject classifications. Primary 30A40.

(C) American Mathematical Society 1972 
(strictly) monotone increasing function of $K$.) The extremal metric for the latter has the form $|Q(z)|^{1 / 2}|d z|$ where $Q(z) d z^{2}$ is a positive quadratic differential in $\Delta_{K}$ with the form $Q(z) d z^{2}=c(z(z-1)(z+K))^{-1} d z^{2}(c$ an appropriate constant). The trajectories of this differential on $\Delta_{K}$ are Jordan curves $\gamma_{t}$ which we may parametrize by their intersections with the positive real axis, $1<t<\infty$. The curve $\gamma_{t}$ divides $\Delta_{K}$ into doublyconnected domains $D_{K t}^{(1)}, D_{K t}^{(2)}$ of which the former is to have the segment $[0,1]$ on its boundary. Let their modules be $M_{K t}^{(1)}, M_{K t}^{(2)}$. There are unique values $r, \rho, 0<r<1,0<\rho<1$, such that $v(r)=M_{K t}^{(1)}, v(\rho)=M_{K t}^{(2)}$ and functions $f_{r, \rho}(z)$ mapping $|z|<1$ conformally onto the interior of $\gamma_{t}$ with $f_{r, \rho}(0)=0, f_{r, \rho}(r)=1$, and $F_{r, \rho}(\zeta)$ mapping $|\zeta|>1$ conformally onto the exterior of $\gamma_{t}$ with $F_{r, \rho}(\infty)=\infty, F_{r, \rho}\left(\rho^{-1}\right)=-K$. For suitable choices of $K, t$ every pair $(r, \rho) \in(0,1) \times(0,1)$ is obtained and the mapping functions are actually determined uniquely by the pair of values $r, \rho$ as follows from the proof of Theorem 1 and is foreshadowed by the notation just given.

THEOREM 1. Let $f(z)$ be regular and univalent in $|z|<1$ with $f(0)=0$. Let $F(\zeta)$ be meromorphic and univalent in $|f|>1$ with a simple pole at the point at infinity. Let the images of $|z|<1$ by $w=f(z)$ and of $|\zeta|>1$ by $w=F(\zeta)$ be nonoverlapping. Then

$$
|f(z) / F(\zeta)| \leqq H\left(|z|,|\zeta|^{-1}\right)
$$

where, $0<r<1,0<\rho<1$,

$$
H(r, \rho)=\left(\mu^{-1}(\nu(r)+\nu(\rho))\right)^{-1} .
$$

Equality is attained when $|z|=r,|\zeta|=\rho^{-1}$, precisely for the pairs of functions $e^{i \varphi} f_{r, \rho}\left(e^{i \alpha} z\right), e^{i \varphi} F_{r, \rho}\left(e^{i \beta} \zeta\right), \alpha, \beta, \varphi$ real, at the points $e^{-i \alpha} r, e^{-i \beta} \rho^{-1}$.

Let $f, F$ satisfy the conditions of Theorem 1 . For specific values $z^{\prime}, \zeta^{\prime}$ with $\left|z^{\prime}\right|=r,\left|\zeta^{\prime}\right|=\rho^{-1}$ consider the images $\varepsilon_{1}, \mathcal{E}_{2}$ of $E_{r}, E_{\rho}$ by $\left(f\left(z^{\prime}\right)\right)^{-1} \times$ $f\left(r z / z^{\prime}\right),\left(f\left(z^{\prime}\right)\right)^{-1} F\left(\rho \zeta^{\prime} z^{-1}\right)$. The doubly-connected domains $\varepsilon_{1}, \varepsilon_{2}$ do not intersect. Let $\mathfrak{D}_{1}, \mathfrak{D}_{2}$ be their (circular) symmetrizations with respect to the positive real axis. These domains have modules at least $v(r), v(\rho)$ and lie in the domain $\Delta_{K^{\prime}}$ with the same topological situation where $K^{\prime}=$ $\left|F\left(\zeta^{\prime}\right)\right| f\left(z^{\prime}\right) \mid$. If then $\mu(K)=v(r)+v(\rho)$ we must have $\mu\left(K^{\prime}\right) \geqq \mu(K)$, i.e., $K^{\prime} \geqq K$. This proves inequality (1). The equality statement follows at once from the standard condition for equality of modules under symmetrization [3].

3. A standard subordination argument makes it possible to drop the condition of univalence in Theorem 1. We will phrase the result for regular functions in the unit circle to correspond to Aharonov's statement. 
CoROllary 1. Let $f(z), g(z)$ be regular for $|z|<1$ with $f(0)=g(0)=0$ and $f(z) g(w) \neq 1$ for arbitrary $z, w$ in the unit circle. Then if $|z|=r$, $|w|=\rho, 0<r, \rho<1$,

$$
|f(z) f(w)| \leqq H(r, \rho) .
$$

Equality is attained precisely for the pairs of functions $e^{i \varphi} f_{r, \rho}\left(e^{i \alpha} z\right)$, $e^{-i \varphi}\left(F_{r, \rho}\left(e^{i \beta} z^{-1}\right)\right)^{-1}, \alpha, \beta, \varphi$ real, at the points $e^{-i \alpha} r, e^{i \beta} \rho$.

We could extend the result to apply also to $z$ or $w$ equal to 0 by stipulating $H(r, 0)=H(0, \rho)=0$. Of course when one variable is zero the extremal functions are not uniquely determined.

The proof of Theorem 1 shows that the assumption that the images under $f$ and $F$ do not overlap is unnecessarily strong. We can state one extension as follows.

CoROllary 2. Let $f(z)$ be regular in $|z|<1$ with $f(0)=0$. Let $F(\zeta)$ be meromorphic in $|\zeta|>1$ with a pole at the point at infinity. Let the images of $|z|<1$ by $w=f(z)$ and of $|\zeta|>1$ by $w=F(\zeta)$ together cover a set of angular measure at most $2 \pi$ on every circle $|w|=R$. Then $(z \neq 0, \zeta \neq \infty)$,

$$
|f(z) / F(\zeta)| \leqq H\left(|z|,|\zeta|^{-1}\right) \text {. }
$$

Equality is attained when $|z|=r,|\zeta|=\rho^{-1}$ precisely for the pairs of functions $e^{i \varphi} f_{r, \rho}\left(e^{i \alpha} z\right), e^{i \varphi} F_{r, \rho}\left(e^{i \beta} \zeta\right), \alpha, \beta, \varphi$ real, at the points $e^{-i \alpha} r, e^{-i \beta} \rho^{-1}$.

This result could also be phrased in the manner of Corollary 1 .

\section{BIBLIOGRAPHY}

1. D. Aharonov, A generalization of a theorem of J. A. Jenkins, Math. Z. 110 (1969), 218-222. MR 40 \#325.

2. James A. Jenkins, On Bieberbach-Eilenberg functions, Trans. Amer. Math. Soc. 76 (1954), 389-396. MR 16, 24.

3. - Some uniqueness results in the theory of symmetrization, Ann. of Math. (2) 61 (1955), 106-115. MR 16, 460.

4. ㄴ, On Bieberbach-Eilenberg functions. III, Trans. Amer. Math. Soc. 119 (1965), 195-215. MR 31 \#5969.

Department of Mathematics, Washington University, St. Louis, Missouri 63130 